Revista de Matemática: Teoría y Aplicaciones 2001 8(1) : 63-76

CIMPA - UCR - CCSS ISSN: 1409-2433

\title{
EXPERIENCIAS CON UN NUEVO ÍNDICE DE FALTA DE AJUSTE EN TABLAS DE CONTINGENCIA
}

\author{
Adalberto GonzÁlez Debén* - Ignacio Méndez Ramírez**
}

Recibido: 8 marzo 2000

\begin{abstract}
Resumen
Rudas, Clogg y Lindsay, en 1994, propusieron un nuevo índice para medir la falta de ajuste de un modelo en tablas de contingencia. Dados una tabla de contingencia $P$, con $N$ celdas, y un modelo $H$, el nuevo índice se define como el valor de $\pi$ para el que se cumple la ecuación$$
P_{h}=(1-\pi) \Pi_{1 h}+\pi \Pi_{2 h}, \quad h=1, \ldots, N
$$

donde $0 \leq \pi \leq 1$, y $\Pi_{1}$ y $\Pi_{2}$ son tablas de probabilidades para cada clase latente. El modelo $H$ se cumple para la primera clase latente y no se hace ninguna suposición para la segunda. El índice de falta de ajuste se interpreta como la proporción de individuos de la población que está intrínsicamente fuera del modelo $H$. En este trabajo se utiliza el análisis de la segunda clase latente para estudiar las causas de la falta de ajuste de un modelo y detectar posibles celdas atípicas Asimismo, se propone utilizar el nuevo índice de falta de ajuste como un concepto de no independencia en el análisis de las frecuencias de las configuraciones de dos muestras.
\end{abstract}

Palabras clave: tabla de contingencia, bondad de ajuste, análisis de residuos, análisis de las frecuenciasde las configuraciones de dos muestras.

\footnotetext{
Abstract

Rudas, Clogg and Lindsay, en 1994, proposed a new index of lack of fit for contingency table analysis. For a contingency table $P$ with $N$ cells, and a model $H$, the new index is defined as the smallest $\pi$ that satisfies the equation

$$
P_{h}=(1-\pi) \Pi_{1 h}+\pi \Pi_{2 h}, \quad h=1, \ldots, N
$$

${ }^{*}$ Centro de Matemática y Física Teórica, Calle E \# 309 esq. a 15, Vedado, Ciudad de La Habana, C.P. 10400, Cuba. Fax: +(537) 3333 73. E-Mail: adal@cidet.icmf.inf.cu

** IIMAS, Universidad Nacional Autónoma de México, Apdo. Postal 20-726 Admon No. 20, Delegación Alvaro Obregón, 01000 México D.F., México. E-Mail: imendez@servidor.unam.mx
} 
with $0 \leq \pi \leq 1$, and $\Pi_{1}$ and $\Pi_{2}$ the tables of probabilities for each latent class. The model $H$ applies to $\Pi_{1}$ but not impose any restrictions on $\Pi_{2}$. The interpretation of the index of lack of fit is the proportion of individuals of the population intrinsically outside model $H$. In this work we propose the analysis of the second latent class to the study of the causes of lack of fit of a model and to detect outlying cells. At the same time, we propose the new index of lack of fit as a concepto of non-independence in the two simple configural frequency analysis.

Keywords: contingency table, goodness of fit, residual analysis, two sample configural frecuency analysis.

Mathematics Subject Classification: : $62 \mathrm{H} 17$

\section{Introducción}

Dada una tabla de contingencia de $N$ celdas, sea $P$ el conjunto de probabilidades en cada una de ellas,

$$
P=\left\{P_{h} ; h=1, \ldots, N\right\}, \quad \sum_{h=1}^{N} P_{h}=1,
$$

sea $f$ el conjunto de las frecuencias observadas,

$$
f=\left\{f_{h} ; h=1, \ldots, N\right\}
$$

y $n$ el tamaño de la muestra,

$$
n=\sum_{h=1}^{N} f_{h}
$$

Sea $e$ el conjunto de las frecuencias esperadas bajo el esquema de muestreo multinomial

$$
e=\left\{e_{h} ; h=1, \ldots, N\right\}
$$

donde

$$
e_{h}=n P_{h}
$$

El procedimiento estándar para comprobar si un cierto modelo $H$ es adecuado es el siguiente:

1. Se obtiene $\hat{e}$, que son las estimaciones máximo verosímiles de los valores esperados bajo el modelo $H$

2. Se comparan $f$ y $\hat{e}$ para decidir si el modelo $H$ se ajusta a los datos.

Las purebas de bondad de ajuste global, como lo indica su nombre, proporcionan una respuesta general a la pregunta si el modelo se ajusta a los datos o no. Sin embargo, no sirven para determinar las causas de falta de ajuste ni tampoco, en caso afirmativo, permiten saber si el modelo realmente representa a todos los datos o si, por el contrario, hay celdas que se desvían considerablemente del valor esperado. Esto sólo se logra observando celda por celda las discrepancias entre el valor observado y el esperado. 


\subsection{Análisis de residuos}

Los residuos estandarizados y eliminados son los más conocidos y utilizados en la literatura.

El residuo estandarizado se define como

$$
r_{h}=\frac{f_{h}-e_{h}}{\sqrt{e_{h}}}
$$

Bajo el supuesto de que el modelo se cumple, $r_{h}$ tiene distribución asintótica normal con media cero.

El residuo eliminado (Simonoff, 1988), es menos conocido. Se trata de la comparación entre el valor observado y el valor perfecto de la celda. El valor perfecto es el que tendría que haber en esa celda para que coincida exactamente con el valor esperado según el modelo de interés y la información de las celdas restantes. El residuo eliminado se define como

$$
r_{h}^{*}=\frac{f_{h}-x}{\sqrt{x}}
$$

donde $x$ es el valor perfecto de la celda $h$.

\subsection{Análisis de las frecuencias de las configuraciones}

El análisis de las frecuencias de las configuraciones (AFC), (Krauth y Lienert, 1973; Von Eye, 1990) es un método no paramétrico desarrollado por G.A. Lienert en 1968 para la búsqueda de tipos y antitipos en tablas de contingencia multidimensionales, con vistas a la determinación de síndromes. Los tipos son combinaciones de las categorías de las variables que ocurren con más frecuencia de lo esperado por azar, mientras que los antitipos son patrones que ocurren con menos frecuencia. En pocas palabras, el AFC consiste en comparar los valores observados y esperados en cada una de las celdas de la tabla de contingencia con el objetivo de determinar si existen diferencias significativas en una o varias de ellas. Estas diferencias significativas sirven como evidencia de la existencia de grupos de infividuos de interés en el sentido de qeu están constituidos por más, o menos, elementos que los esperados bajo un cierto modelo. Aunque el AFC es una técnica típicamente exploratoria, se puede utilizar con fines confirmatorios en una etapa más avanzada cuando lo que interesa en analizar sólo un subconjunto de celdas; que pueden haber sido identificadas en un estuido exploratorio anterior con otro conjunto de datos o derivarse del conocimiento sustantivo.

"La prueba más conocida y más ampliamente utilizad en el AFC es la $X^{2}$ de Pearson" (von Eye et al, 1996). Para la celda $h$ de una tabla de contingencia con $N$ celdas se tiene

$$
X_{h}^{2}=\frac{\left(f_{h}-e_{h}\right)^{2}}{e_{h}}
$$

Como en el AFC exploratorio se realizan simultáneamente $N$ pruebas de hipótesis, se requiere proteger el nivel de significación $\alpha$. Las dos estrategias más utlizadas para este propósito son el ajuste de Bonferroni $\left(\alpha^{*}=\frac{\alpha}{N}\right)$, y el ajuste de Holm $\alpha_{i}^{*}=\frac{\alpha}{N-i+1}, i=$ $1, \ldots, N$. Este último es menos conservador y presupone que las pruebas están ordenadas. 
La interpretación de los tipos y antitipos depende del modelo nulo que se utiliza para calcular los valores esperados. Los modelos nulos tienen la característica de que consideran todas las relaciones entre las variables excepto algunas de interés. Cuando el modelo nulo se rechaza, se debe a que sí existen las relaciones no consideradas en el modelo. En el AFC no interesa el valor numérico del ajuste global del modelo nulo, ni interpretar sus parámetros o encontrar un modelo más complicado que se ajuste, sino detectar los tipos $\mathrm{y}$ antitipos e interpretarlos a la luz del modelo nulo correspondiente.

\section{Indice de falta de ajuste de Fudas, Clogg y Lindsay}

Rudas et al (1994), propusieron un nuevo índice de falta de ajuste (NIFA) que sirve para cualquier tabla de contingencia y cualquier modelo. Dados una tabla de contingencia $P$, con $N$ celdas y un modelo $H$, se propone una familia de modelos $\left\{H_{\pi}\right\}$ de la forma

$$
P_{h}=(1-\pi) \Pi_{1 h}+\pi \Pi_{2 h}, \quad h=1, \ldots, N ; \quad 0 \leq \pi \leq 1
$$

Se trata de expresar la tabla $P$ como una combinación convexa de dos subtablas $\Pi_{1}$ y $\Pi_{2}$, también llamadas primera cara y segunda cara respectivamente, de manera que el modelo $H$ se comple para la subtabla $\Pi_{1}$ y no se hace ninguna suposición respecto a la subtabla $\Pi_{2}$. La ecuación (1) es una generalización del modelo $H$, pues representa una familia que va desde el modelo $H(\pi=0)$ hasta el modelo completamente irrestricto $(\pi=1)$. Por otro lado, al no incluir ninguna suposición para la segunda subtabla, constituye una generalización del modelo usual de clases latentes (que supone el modelo de independencia para ambas tablas).

Se define el índice de falta de ajuste, $\pi^{*}$, como el menor valor de $\pi$ para el que se cumple la ecuación (1). La interpretación de $\pi^{*}$ es muy sencilla: es la proporción de individuos de la población que está intrínsicamente fuera del modelo $H$ (medida con error o mal clasificada). Para fines prácticos, se propone como una medida de la falta de ajuste del modelo $H$, que describe la magnitud de las interacciones de orden superior que no se contemplan en el mismo.

Como no se le impone ninguna restricción a la subtabla $\Pi_{2}$, se supone que en ella se concentra la parte de la población que no es modelada por $H$. Si $\pi^{*}$ resulta grande, y por lo tanto se requiere mejorar el modelo $H$, se puede inspeccionar esta subtabla y de esta manera obtener información útil para este propósito. Esto se considera una alternativa al análisis de residuos en el procedimiento usual.

\begin{tabular}{|c|c|c|c|}
\hline Filas/columnas & Columna 1 & Columna 2 & Total \\
\hline Fila 1 & $A$ & $B$ & $A+B$ \\
\hline Fila 2 & $C$ & $D$ & $C+D$ \\
\hline Total & $A+C$ & $B+D$ & $n$ \\
\hline
\end{tabular}

Cuadro 1: Frecuencias observadas en una tabla de $2 \times 2$.

En el caso de dos variables dicotómicas se puede obtener una fórmula explícita para $\pi^{*}$. La tabla 1 representa las frecuencias observadas. 
Considerando $A D-B C>0$ y $A>D$, el valor perfecto de la celda correspondiente a la fila 2 , columna 2 , es

$$
x=\frac{B C}{A}
$$

luego

$$
\pi^{*}=\frac{A D-B C}{A n}
$$

Por último, la descomposición en dos caras queda de la siguiente manera:

$$
\left[\begin{array}{ll}
A & B \\
C & D
\end{array}\right]=\left[\begin{array}{cc}
A & B \\
C & \frac{B C}{A}
\end{array}\right]+\left[\begin{array}{cc}
0 & 0 \\
0 & D-\frac{B C}{A}
\end{array}\right]
$$

Nótese que cuando $A=D$, si bien la expresión de $\pi^{*}$ no cambia, se pueden obtener dos descomposiciones equivalentes de la tabla original dependiendo de la celda a la que se le asigne el valor perfecto.

\section{Detección de celdas atípicas (outliers)}

Según Barnett y Lewis (1994), "En sentido general, un outlier en un conjunto de datos es una observación que parece inconsistente con el modelo supuesto". En el caso de tablas de contingencia este concepto se refiere a las celdas y no a los individuos aislados.

\subsection{Análisis usual}

Haberman (1973) propuso inspeccionar gráficamente los residuos ajustados. Brown (1974) propuso declarar una celda como atípica si el modelo de independencia no se ajusta y el de cuasi-independencia, que resulta de considerar esa celda como cero estructural, sí se ajusta. Fuchs y Kenet (1980) propusieron un test con este mismo fin, y Simonoff (1988) otro que lo supera en cuanto a la capacidad para detectar más de una celda. Otros autores han explorado la conveniencia de aplicar conceptos ya establecidos con el contexto de análisis de regresión como son los de palanca (Andersen, 1992; Upton y Guillen, 1995) e influencia (Upton y Guillén, 1995).

El consenso parece ser favorable al residuo elimando $r^{*}$ y a la diferencia del estadígrafo de razón de verosimilitud

$$
\Delta G^{2}=G^{2}-G_{c}^{2}
$$

donde $G^{2}$ es el estadígrafo de razón de verosimilitud para la tabla original y $G_{c}^{2}$ es el estadígrafo de razón de verosimilitud que resulta de sustituir $f_{h}$ por $x$.

Cuando sólo se cuenta con la información de que un cierto modelo no se ajusta, se deben hacer tantas pruebas como celdas tiene la tabla. Para poder garantizar que la probabilidad de cometer el error de tipo I no exceda el nivel de significación prefijado $\alpha$, se debe tomar un nivel tal que permita que esto no suceda. Por ejemplo, $\alpha^{*}=\frac{\alpha}{N}$, donde $N$ es el número de celdas.

Para ilustrar la utilización de $r, r^{*}$ y $\Delta G^{2}$ se muestran dos ejemplos muy conocidos en la literatura acerca de esta temática. El primer ejemplo, (Wickens, 1989), es una tabla de 
$3 \times 4$ con una celda atípica. El segundo ejemplo, (Simonoff, 1988; Barnett y Lewis, 1994) es una tabla de $5 \times 5$ con tres celdas atípicas. En todos lo casos se trabajó con el modelo de independencia.

\section{Ejemplo 1 Una celda atipica}

En la tabla 2 a se muestran los datos que analiza Wickens (1989), y en las tablas 2b, $2 c$ y $2 d$ se muestran resultados.

\begin{tabular}{|c|c|c|c|}
\hline 12 & 18 & 3 & 10 \\
\hline 18 & 3 & 7 & 10 \\
\hline 20 & 6 & 9 & 11 \\
\hline
\end{tabular}

Tabla 2.a Tabla 10.4, página 252, Wickens(1989)

\begin{tabular}{|c|c|c|c|}
\hline$-1,2$ & $\mathbf{2 . 9}$ & $-1,4$ & $-0,2$ \\
\hline 0,8 & $-1,8$ & 0,6 & 0,2 \\
\hline 0,4 & $-1,2$ & 0,8 & $-0,1$ \\
\hline
\end{tabular}

Tabla 2.b Residuos estandarizados

\begin{tabular}{|c|c|c|c|}
\hline$-2,68$ & $\mathbf{8 . 6 6}$ & $-2,09$ & $-0,3$ \\
\hline 2,02 & $-2,76$ & 0,99 & 0,46 \\
\hline 1,2 & $-2,14$ & 1,66 & $-0,14$ \\
\hline
\end{tabular}

Tabla 2.c Residuos eliminados para cada una de las celdas de la tabla 2a.

\begin{tabular}{|c|c|c|c|}
\hline 3.67 & $\mathbf{1 5 . 7 4}$ & 3.63 & 0.047 \\
\hline 1.44 & 6.66 & 0.49 & 0.11 \\
\hline 0.51 & 3.08 & 1.17 & 0.0096 \\
\hline
\end{tabular}

Tabla 2.d $\Delta G^{2}$ para cada una de las celdas de la tabla 2a.

Para $\alpha^{*}=\frac{0,05}{12}=0,00416$ el valor crítico de la distribución ji-cuadrado con un grado de libertad es 8.21. Como conclusión resulta evidente que según todos los puntos de vista considerados se detecta la celda (1,2) como atípica.

\section{Ejemplo 2 Varias celdas atípicas}

En la tabla 3a, se muestran los datos que analizan Simonoff (1988), y Barnett y Lewis (1994), y en las tablas 3b, 3c y 3d se muestran los resultados.

Los resiudos de las celdas (1,1), (1,2), (1,3) y (1,4) son los más grandes. Para $\alpha^{*}=$ $\frac{0,05}{25}=0,02$ el valor crítico de la distribución ji-cuadrado es 9.55. Según el criterio $\Delta G^{2}$ sólo se detecta la celda (1,1) como atípica.

Como conclusión se puede decir que:

1. Todos estos criterios son efectivos cuando hay una sola celda atípica. 


\begin{tabular}{|l|l|l|l|l|}
\hline 18 & 41 & 41 & 20 & 21 \\
\hline 39 & 20 & 20 & 22 & 22 \\
\hline 24 & 20 & 20 & 16 & 18 \\
\hline 20 & 20 & 19 & 19 & 19 \\
\hline 23 & 19 & 20 & 17 & 20 \\
\hline
\end{tabular}

Tabla 3.a Tabla 10.4. página 252, Simonoff (1988), y Barnett y Lewis (1994)

\begin{tabular}{|c|c|c|c|c|}
\hline $\mathbf{- 2 . 3 8}$ & $\mathbf{1 . 9 4}$ & $\mathbf{1 . 9 4}$ & -0.77 & -0.85 \\
\hline $\mathbf{2 . 2 3}$ & -1.25 & -1.25 & 0.28 & -0.009 \\
\hline 4.8 & -0.23 & -0.23 & -0.13 & 0.1 \\
\hline-0.34 & -0.19 & -0.41 & 0.66 & 0.39 \\
\hline 0.21 & -0.5 & -0.28 & 0.08 & 0.54 \\
\hline
\end{tabular}

Tabla 3.b Residuos estandarizados

\begin{tabular}{|c|c|c|c|c|}
\hline $\mathbf{- 3 . 6 9}$ & $\mathbf{3 . 6 5}$ & $\mathbf{3 . 6 4}$ & -1.19 & -1.33 \\
\hline $\mathbf{4 . 1 1}$ & -1.94 & -1.94 & 0.44 & -0.014 \\
\hline 0.76 & -0.36 & -0.36 & -0.18 & 0.15 \\
\hline-0.51 & -0.29 & -0.62 & 0.99 & 0.58 \\
\hline 0.34 & -0.75 & -0.43 & 0.12 & 0.81 \\
\hline
\end{tabular}

Tabla 3.c Residuos eliminados para cada una de las celdas de la tabla 3.a.

\begin{tabular}{|c|c|c|c|c|}
\hline 10.66 & 6.12 & 6.12 & 0.98 & 1.21 \\
\hline 7.74 & 2.7 & 2.7 & 0.12 & 0.00 \\
\hline 0.35 & 0.085 & 0.085 & 0.023 & 0.016 \\
\hline 0.18 & 0.06 & 0.26 & 0.61 & 0.22 \\
\hline 0.07 & 0.39 & 0.12 & 0.01 & 0.42 \\
\hline
\end{tabular}

Tabla 3.d $\Delta G^{2}$ para cada una de las celdas de la tabla 3a.

2. Cuando hay más de una celda atíica puede que alguna quede oculta o que alguna, que en realidad no lo es, lo parezca.

\subsection{Una alternativa al análisis de residuos usual}

En este acápite se propone el análisis de la "segunda cara de Rudas et al" como otra forma de estudiar las causas de la falta de ajuste de un moedlo. A modo de ilustración se consideran los dos ejemplos ya vistos en el epígrafe anterior.

En el primer ejemplo (Wickens, 1989), el $23 \%$ de los individuos se aparta de la hipótesis verdadera de independencia y más de la mitad de ellos se concentra en la celda $(1,2)$. En la tabla 4 se muestran los resultados.

El segundo ejemplo es la tabla presentada por Simonoff (1984) y Barnett y Lewis (1994). Los resultados se muestran en las tablas 5a y 5b. 


\begin{tabular}{|c|c|c|c|c|}
\hline Celda & Observado & cara 1 & cara 2 & cara 2(\%) \\
\hline 1.1 & 12 & 11.95 & 0.01 & 0.05 \\
\hline 1.2 & 18 & 2.11 & $\mathbf{1 5 . 7 5}$ & $\mathbf{5 3 . 9 2}$ \\
\hline 1.3 & 3 & 3.05 & 0.00 & 0.00 \\
\hline 1.4 & 10 & 6.66 & 3.26 & 11.17 \\
\hline 2.1 & 18 & 17.2 & 0.76 & 2.58 \\
\hline 2.2 & 3 & 3.03 & 0.0 & 0.00 \\
\hline 2.3 & 7 & 4.39 & 2.56 & 8.76 \\
\hline 2.4 & 10 & 9.58 & 0.38 & 1.31 \\
\hline 3.1 & 20 & 19.8 & 0.15 & 0.5 \\
\hline 3.2 & 6 & 3.49 & 2.46 & 8.43 \\
\hline 3.3 & 9 & 5.05 & 3.88 & 13.27 \\
\hline 3.4 & 11 & 11.02 & 0.00 & 0.02 \\
\hline Total & 127 & 95.25 & 25.75 & 100.0 \\
\hline
\end{tabular}

Cuadro 4: Análisis de la segunda cara de Rudas para la tabla 2a.

Como se puede apreciar, en la segunda cara se destacan las celdas $(1,2)(1,3)$ y $(2,1)$.

\section{AFC de varias muestras}

En el AFC de $k$ muestras se plantea como hipótesis nula que todos los individuos provienen de la misma población. El modelo nulo correspondiente es un modelo loglineal saturado para el grupo de variables predictoras, que a su vez son independientes de la variable que representa a los grupos. Por ejemplo, si se tienen las variables discriminantes $A$ y $B$ y además la variable $G$ que representa a los grupos, con índices $i, j$ y $g$ respectivamente, el modelo tienen la forma

$$
\log \left(P_{i j g}\right)=\lambda+\lambda_{A(i)}+\lambda_{B(i)}+\lambda_{G(g)}
$$

En el AFC de dos muestras, siempre que se detecta un tipo, también se detecta un antitipo en la celda aledaña pues la suma de los valores esperados de ambas celdas es igual a la de los valores observados. Por eso, en lugar de hacer referencia a cada una de las dos celdas, se menciona genéricamente la combinación de características explicatorias como "configuración problacionalmente diferente".

En una segunda etapa del análisis se comparan los grupos en cada configuración. Para ello se toman en cuenta las subtablas donde las columnas representan a los grupos y las filas a la configuración que se analiza y el resto de las configuraciones agrupadas. Cada una de estas tablas se puede analizar por separado. En la tabla 6 se ilustra este procedimiento para el caso de dos poblaciones.

Como ejemplo se presenta el de los suicidios en Alemania (Krauth y Lienert, 1973; von Eye, 1990). Lo que interesa saber es si los suicidas de ambos sexos recurren a los mismos métodos para autoeliminarse y si esto depende o no del tiempo transcurrido desde la II Guerra Mundial. Para ello se toman los datos de suicidios de los años 1944 y 1952 


\begin{tabular}{|c|c|c|c|c|}
\hline 18.14 & 20.97 & 20.98 & 18.27 & 20.13 \\
\hline 17.29 & 19.99 & 20 & 17.42 & 19.19 \\
\hline 15.89 & 18.37 & 18.37 & 16 & 17.63 \\
\hline 16.32 & 18.87 & 18.87 & 16.44 & 18.11 \\
\hline 16.33 & 18.89 & 18.89 & 16.45 & 18.12 \\
\hline
\end{tabular}

Tabla 5.a Método de Ruda para la tabla 3a y modelo de independencia (cara 1).

\begin{tabular}{|c|c|c|c|c|}
\hline 0.0 & $\mathbf{1 9 . 9 2}$ & $\mathbf{1 9 . 9 1}$ & 1.67 & 0.84 \\
\hline $\mathbf{2 1 . 6 1}$ & 0.05 & 0.04 & 4.53 & 2.77 \\
\hline 8.05 & 1.56 & 1.56 & 0.1 & 0.4 \\
\hline 3.63 & 1.09 & 0.2 & 2.52 & 0.9 \\
\hline 6.6 & 0.18 & 1.06 & 0.54 & 1.85 \\
\hline
\end{tabular}

Tabla 5.b Método de Rudas para la tabla 3a y el modelo de independencia (cara 2).

\begin{tabular}{|c|c|c|c|}
\hline Configuraciones $A B$ & Muestra 1 & Muestra 2 & Total \\
\hline Celda $(i j)$ & $f_{i j 1}$ & $f_{i j 2}$ & $f_{i j 1}-f_{i j 2}$ \\
\hline Demás configuraciones & $N_{1}-f_{i j 1}$ & $N_{2}-f_{i j 2}$ & $N-f_{i j 1}-f_{i j 2}$ \\
\hline Total & $N_{1}$ & $N_{2}$ & $N$ \\
\hline
\end{tabular}

Cuadro 6: Análisis de al configuración $(i j)$.

considerando ambos sexos y siete métodos (gas, cuerda, soporífero, ahogado, venas, disparo y salto). Se tomó un $\alpha=0,05$ con ajuste de Bonferroni $\left(\alpha^{*}=\frac{0,05}{14}=0,00357\right)$. En la tabla 7 se muestran los resultados.

Se puede apreciar que hay seis tipos discriminatorios. En 1952 las mujeres se suicidaban utilizando soporíferos más frecuentemente que los hombres y los hombres se cortaban las venas más frecuentemente que las mujeres. En 1944 los métodos de suicidio por gas y ahogado fueron más frecuentes en las mujeres que en los hombres mientras que los de ahorcarse y dispararse fueron más frecuentes en los hombres que en las mujeres.

\subsection{Desarrollos más recientes del AFC}

Desde un inicio, los críticos del AFC argumentaron que no era más que un análisis de residuos en el contexto de lso modelos loglineales. Los defensores del AFC destacan que es un método para la investigación diferencial, por lo que más que para comprobar hipótesis globales acerca de la relación entre variables, está dirigido a estudiar las celdas que se desvían de ciertas hipótesis locales referidas a la relación entre las variables. Por otra parte, como el análisis de residuos está supeditado a la selección de modelos, se puede utilizar para cualquier modelo más sencillo que el saturado. Sin embargo, para el AFC sólo resulta de interés un subconjunto de los modelos loglineales.

Victor (1989) y Keiser y Victor (1991) desarrollaron un enfoque alternativo al AFC estándar basado en el modelo loglineal de cuasi-independencia. La fundamentación se basa en que si se supone que los individuos de una celda no provienen de la misma población 


\begin{tabular}{|l|cc|cc|cc|}
\hline Configuración & \multicolumn{2}{|c|}{ Observados } & \multicolumn{2}{c|}{ Esperados } & \multicolumn{2}{c|}{ Significancia } \\
\hline (Año, método) & Mascullino & Femenino & Masculino & Femenino & $\chi^{2}$ & $p\left(\chi^{2} ; g l=1\right)$ \\
\hline 1952, gas & 52 & 47 & 49.5 & 49.5 & 0.364 & 0.4537 \\
\hline 1952, cuerda & 31 & 14 & 22.5 & 22.5 & 7.071 & 0.0078 \\
\hline 1952, soporífico & 44 & 97 & 70.5 & 70.5 & 24.180 & $\prec \alpha^{*}$ \\
\hline 1952, ahogado & 20 & 10 & 15.0 & 15.0 & 3.602 & 0.0577 \\
\hline 1952, venas & 22 & 5 & 13.5 & 13.5 & 11.335 & $\prec \alpha^{*}$ \\
\hline 1952, disparo & 3 & 0 & 1.5 & 1.5 & 3.047 & 0.0809 \\
\hline 1952, salto & 2 & 2 & 2.0 & 2.0 & 0 & 1.0 \\
\hline 1944, gas & 16 & 61 & 38.5 & 38.5 & 28.967 & $\prec \alpha^{*}$ \\
\hline 1944, cuerda & 76 & 35 & 55.5 & 55.5 & 18.548 & $\prec \alpha^{*}$ \\
\hline 1944, soporífero & 7 & 9 & 8.0 & 8.0 & 0.233 & 0.6293 \\
\hline 1944, ahogado & 19 & 54 & 36.5 & 36.5 & 18.287 & $\prec \alpha^{*}$ \\
\hline 1944, venas & 15 & 4 & 9.5 & 9.5 & 6.675 & 0.0098 \\
\hline 1944, disparo & 35 & 11 & 23.0 & 23.0 & 133.695 & $\prec \alpha^{*}$ \\
\hline 1944, salto & 9 & 2 & 5.5 & 5.5 & 4.604 & 0.0318 \\
\hline
\end{tabular}

Cuadro 7: Métodos de suicidio en Alemania según sexo y año. (Krauth y Lienert, 1973; von Eye, 1990)

que el resto, es inapropiado estimar los valores esperados de toda la tabla utilizando la informaión de esa celda. De acuerdo con este planteamiento, las celdas que constituyen posibles tipos o antitipos se consideran ceros estructurales. El procedimiento consta de dos pasos: primero se ajusta el modelo de cuasi-independencia y luego, si se cumple la independencia en la tabla reducida, se estiman las frecuencias en las celdas objeto de análisis y se comparan los valores observados.

Goodman (1991) propone tres medidas de no independencia para tablas de contingencia de dos entradas: la medida de diferencia relativa $(\Delta)$, la medida de interacción no ponderada $(\lambda)$ y la medida de interacción ponderada $(\lambda)$. Estas medidas para el caso de una tabla de $2 x 2$ con probabilidades $P_{11}, P_{12}, P_{21}$ y $P_{22}$, están relacionadas con la razón de productos cruzados $(\theta)$ y el coeficiente de correlación $(\rho)$

$$
\begin{gathered}
\Delta=\rho \\
\lambda=\frac{\log (\theta)}{4} \\
\lambda=\log (\theta) \sqrt{P_{1+} P_{2+} P_{+1} P_{+2}}
\end{gathered}
$$

Von Eye et al. (1995) propone utilizar las tres medidas de no independencia de Goodman (1991) para definir nuevos conceptos alternativos de tipo en el AFC de varias muestras. Para ejemplificar su procedimiento utilizó el mismo ejemplo de los suicidos en Alemania. En la tabla 8 se reproducen los resultados de este trabajo de manera simplificada, pues sólo se señalan los resultados significativos.

Este trabajo constituye un nuevo enfoque en el AFC exploratorio y deja abierta la posibilidad de incorporar nuevas medidas de no independencia a la definición de tipos y 


\begin{tabular}{|l|c|c|c|c|}
\hline $\begin{array}{l}\text { Configuración } \\
\text { (Año, método) }\end{array}$ & $p(\lambda)$ & $p(\lambda)$ & $p(\rho)$ & $p(\theta)$ \\
\hline 1952, gas & & & & $*$ \\
\hline 1952, cuerda & & & & $*$ \\
\hline 1952, soporífico & $*$ & $*$ & $*$ & $*$ \\
\hline 1952, ahogado & & & & \\
\hline 1952, venas & $*$ & $*$ & $*$ & \\
\hline 1952, disparo & $*$ & & & $*$ \\
\hline 1952, salto & & & & \\
\hline 1944, gas & $*$ & $*$ & $*$ & $*$ \\
\hline 1944, cuerda & $*$ & $*$ & $*$ & $*$ \\
\hline 1944, soporífico & & & & \\
\hline 1944, ahogado & $*$ & $*$ & $*$ & $*$ \\
\hline 1944, venas & & & & \\
\hline 1944, disparo & $*$ & $*$ & $*$ & $*$ \\
\hline 1944, salto & & & & \\
\hline
\end{tabular}

Cuadro 8: Tipos detectados con cada una de las medidas de no independencia de Goodman (von Eye et al. 1995).

antitipos con vistas a disponer de diferentes perspectivas de la desvación con respecto a la independencia.

\subsection{Un nuevo enfoque de tipo en el análisis de las frecuencias de las configuraciones}

En este trabajo se porpone el análisis de la segunda cara como un análisis exploratorio con vistas a sugerir la posible existencia de tipos, factibles de confirmar en una etapa posteriore mediante un análisis confirmatorio dirigido a este fin.

Siguiendo el espíritu del trabajo de von Eye at al. (1995), que propone nuevos conceptos de tipo a partir de las diferentes medidas de no independencia dadas por Goodman (1991), en este trabajo se propone utiizar el NIFA como un nuevo concepto de no independencia para identificar configuraciones poblacionalmente diferentes en el AFC de dos muestras.

Para ilustrar esta utilización del NIFA se utilizó el mismo ejemplo presentado por Krauth y Lienert (1973), von Eye (1990) y von Eye at al (1995). Se encontró que aproximadamente el $40 \%$ de los individuos no concuerda con la hipótesis de que los individuos provienen de la misma población. En la tabla 9 se muestran los resultados de las dos caras del modelo de Rudas et al.

Como se puede apreciar, las celdas 5 que más contribuyen al $40 \%$ que no se ajusta están incluidas dentro de los seis tipos que se detectaron en el AFC de dos poblaciones estándar. Estos son los correspondientes a las configuraciones: "1952, soporífero", "1944, gas", "1944, cuerda", "1944, ahogado" y "1944, disparo".

Nótese cómo los individuos que se encuentran en las celdas con menor valor observado 


\begin{tabular}{|c|c|c|c|c|c|c|}
\hline \multirow{2}{*}{$\begin{array}{l}\text { Configuración } \\
\text { (Año, método) }\end{array}$} & \multicolumn{2}{|c|}{ Observados } & \multicolumn{2}{|c|}{ Esperados } & \multicolumn{2}{|c|}{ Significancia } \\
\hline & Mascullino & Femenino & Masculino & Femenino & $\chi^{2}$ & $p\left(\chi^{2} ; g l=1\right)$ \\
\hline 1952, gas & 32.95 & 46.14 & 18.89 & 0.8 & 6.66 & 0.28 \\
\hline 1952, cuerda & 9.8 & 13.73 & 21.1 & 0.25 & 7.44 & 0.09 \\
\hline 1952, soporífico & 43.86 & 61.43 & 0.1 & 35.28 & 0.04 & 12.43 \\
\hline 1952,ahogado & 6.82 & 9.55 & 13.12 & 0.44 & 4.62 & 0.15 \\
\hline 1952, venas & 3.52 & 4.93 & 18.41 & 0.07 & 6.49 & 0.02 \\
\hline 1952, disparo & 0.00 & 0.00 & 2.99 & 0.00 & 1.05 & 0.00 \\
\hline 1952, salto & 0.55 & 0.76 & 1.45 & 1.23 & 0.51 & 0.43 \\
\hline 1944, gas & 15.96 & 22.36 & 0.02 & 38.46 & 0.01 & 13.55 \\
\hline 1944, cuerda & 24.88 & 34.84 & 50.88 & 0.11 & 17.93 & 0.04 \\
\hline 1944, soporífero & 4.41 & 6.18 & 2.57 & 2.8 & 0.91 & 0.99 \\
\hline 1944, ahogado & 18.90 & 26.47 & 0.08 & 27.37 & 0.0 & 9.64 \\
\hline 1944, venas & 2.75 & 3.85 & 12.2 & 0.14 & 4.3 & 0.05 \\
\hline 1944, disparo & 7.78 & 10.89 & 27.12 & 0.09 & 9.56 & 0.03 \\
\hline 1944, salto & 1.33 & 1.86 & 7.64 & 0.14 & 2.69 & 0.05 \\
\hline
\end{tabular}

Cuadro 9: Método de Rudas et al aplicado a la tabla 7.

dentro de cada fila se ubican en la cara 1; mientras que los infidividuos que se alejan de la hipótesis de independencia son una parte de los que se encuentran en las celdas con mayor valor observado dentro de cada fila.

En la tabla 10 se repite la tabla 8, con los resultados de los nuevos conceptos de tipo propuestos por von Eye et al (1995). A esta tabla se le añadió una columna donde se muestra el valor del NIFA.

Como se puede apreciar, las configuraciones (1952, soporífero), (1944, gas), (1944, cuerda), (1944, ahogado) y (1944, disparo), en ese orden, son las que tienen mayores valores de $\pi^{*}$. Estas mismas configuraciones son las que resultan poblacionalmente diferentes según los cuatro criterios de von Eye et al (1995): $\lambda, \lambda, \rho$ y $\theta$.

\section{Conclusiones}

El análisis de la segunda cara sirve como herramienta más para estuidar las causas de falta de ajuste de un modelo y detectar posibles celdas atípicas. Con ese fin se puede utilizar como complemento del análissi de residuos estandarizados, el análisis de residuos eliminados y la variación del estadístico de razón de verosimilitud.

La utilización de este método en el análisis de las frecuencias de las configuraciones es una idea afín al enfoque de Victor (1989) y Kieser y Victor (1991), pues en la primera cara quedan "todas las celdas perfectas" y la segunda contiene a los "individuos que sobran".

En el caso de una tabla de 2x2 el valor del índice de falta de ajuste coincide exactamente con una de las diferencias entre el valor observado y el valor de cada celda perfecta (expresado como proporción de la muestra total). Debido a esta coincidencia, resulta natural la utilización de este nuevo criterio no sólo como una ayuda a la interpretación de 


\begin{tabular}{|c|c|c|c|c|c|}
\hline $\begin{array}{c}\text { CONFIGURACIÓN } \\
\text { (Año, método) }\end{array}$ & $p(\lambda)$ & $p(\lambda)$ & $p(\rho)$ & $p(\theta)$ & $\pi^{*}(\%)$ \\
\hline 1952, gas & & & & $*$ & 0.82 \\
\hline 1952, cuerda & & & & $*$ & 2.52 \\
\hline 1952, soporífico & $*$ & $*$ & $*$ & $*$ & 8.63 \\
\hline 1952, ahogado & & & & & 1.46 \\
\hline 1952, venas & $*$ & $*$ & $*$ & & 2.45 \\
\hline 1952, disparo & $*$ & & & $*$ & 0.43 \\
\hline 1952, salto & & & & & 0.0 \\
\hline 1944, gas & $*$ & $*$ & $*$ & $*$ & 6.71 \\
\hline 1944, cuerda & $*$ & $*$ & $*$ & $*$ & 6.48 \\
\hline 1944, soporífico & & & & & 0.29 \\
\hline 1944, ahogado & $*$ & $*$ & $*$ & $*$ & 5.27 \\
\hline 1944, venas & & & & & 1.58 \\
\hline 1944, disparo & $*$ & $*$ & $*$ & $*$ & 3.52 \\
\hline 1944, salto & & & & & 1.1 \\
\hline
\end{tabular}

Cuadro 10: Tipos detectados con las medidas de no independencia de Goodman y valor del NIFA.

los tipos y antitipos en el AFC de dos poblaciones, sino como un concepto alternativo de tipos.

\section{Recomendaciones}

En el trabajo original de Rudas et al (1994), se recomienda estudiar el caso de los ceros muestrales; esto debe extenderse al caso más general de celdas subfrecuentes.

Debe señalarse que, en el contexto del AFC estándar, no queda claro cómo identificar los antitipos con el análisis de la segunda cara de Rudas; pues este enfoque se ocupa de los individuos que se alejan de un modelo dado y no de los que faltan para que dicho modelo se cumpla. Una posible ampliación de las posibilidades de este enfoque es utilizar la diferencia entre la falta de ajuste del modelo de independencia y la falta de ajuste del modelo de cuasi-independencia que resulta de considerar a las celdas objeto de análisis como si fueran ceros estructurales, para describir dicho conjunto de celdas sin importar si se trata de tipos o antitipos.

Se necesita seguir experimentando esta metodología con otros ejemplos de la literatura y problemas reales, que involucren más variables y modelos más complejos.

\section{Referencias}

[1] Agresti, A. (1990) Categorical Data Analysis. Wiley, New York.

[2] Andersen, E.B. (1992) "Diagnostic in categorical data analysis", J.R.S.S. B54: 781-791. 
[3] Barnett, V.D.; Lewis, T. (1994) Outliers in Statistical Data, $3^{\text {rd }}$ Ed. Wiley, New York.

[4] Brown, M.B. (1974) "Identification of the sources of significance in two-way contingency tables", Applied Statistics 23: 405-413.

[5] Fuchs, C.; Kenett, R. (1980) "A test for detecting outlying cells in the multinomial distribution and two-way contingency tables", JASA 75 395-398.

[6] Goodman, L.A. (1991) "Measures, models and graphical displayas in the analysis of crossclassified data", JASA 86: 1085-1111.

[7] González, A.D. (1996) "Algo más acerca de la falta de ajuste de un modelo en el análisis de tablas de contingencia", ponencia presentada en el VI Encuentro de Estadística Cuba-México, La Habana.

[8] Haberman, S.J. (1973) "The analysis of residual in cross-classified tables", Biometrics 29: $205-220$.

[9] Kieser, M.; Victor, N. (1991) "A test procedure for an alternative approach to configural frecuency analysis", Methodika 5. 87-97.

[10] Krauth, J.; Lienert, G.A. (1973) Die Konfigurationsfrequenzanalyse und ihre Anwendung in Psychologie und Medizin. Alber, Freiburg.

[11] Little, R.F.A.; Rubin, B. (1987) Statistical Analysis with Missing Data. New York: Wiley.

[12] Montero, M.D.; González, A.D.; Vidaillet, J. (1994) "Uso de los modelos jerárquicos loglineales y logísticos en el análsis de las frecuencias de las configuraciones de dos muestras", Ciencias Técnicas, Físicas y Matemáticas 15: 77-88.

[13] Rudas, T.; Clogg, C.C.; Lindsay, B.G. (1994) "A new index of fit based on mixture methods for the analysis of contingency tables", J.R.S.S. B56: 623-639.

[14] Simonoff, T.S. (1988) "Detecting outlying cells in two-ways contingency tables via backwardsstepping", Technometrics 30: 339-345.

[15] Upton, G.J.G.; Guillén, M. (1995) "Perfect cells, direct models and contingency table outliers", Commun. Statist.-Theory Meth., 24: 1843-1862.

[16] Victor, N. (1989) "An alternative approach to configural frecuency analysis", Methodika III: $61-73$.

[17] von Eye, A. (1990) Introduction to Configural Frecuency Analysis: The search for Types and Antitypes in Cross-Classiffications. Cambridge University Press.

[18] von Eye, A.; Spiel, C.; Rovine, M.J. (1995) "Concepts of nonindependence in Configural Frecuency Analysis", Journal of Mathematical Sociology 20: 41-54.

[19] von Eye, A.; Spiel, C.; Wood, P.K. (1996) "Configural frecuency analysis in applied psychological research", Applied Psychology: an International Review 45: 301-352.

[20] Wickens, T.D. (1989) Multivariate contingency tables analysis for the behavioral sciences. Lawrence Erlbaum Associates Inc., Hillsdale, N.J. 